Article

\title{
Dissipative Dynamics of Non-Interacting Fermion Systems and Conductivity
}

\author{
Kazuki Yamaga (D) \\ Department of Nuclear Engineering, Kyoto University, Kyoto 606-8501, Japan; \\ yamaga.kazuki.62a@st.kyoto-u.ac.jp
}

Received: 11 September 2020; Accepted: 31 October 2020; Published: 3 November 2020

\begin{abstract}
In this paper, Non-Equilibrium Steady State that is induced by electric field and the conductivity of non-interacting fermion systems under the dissipative dynamics is discussed. The dissipation is taken into account within a framework of the quantum dynamical semigroup introduced by Davies (1977). We obtain a formula of the conductivity for the stationary state, which is applicable to arbitrary potentials. Our formula gives a justification of an adiabatic factor that is often introduced in practical calculation while using the Kubo formula. In addition, the conductivity of crystals (i.e., periodic potentials) is also discussed.
\end{abstract}

Keywords: open quantum system; non-equilibrium physics; statistical mechanics; fermion system; electric conductivity

\section{Introduction}

Given a macroscopic quantum system, its thermodynamical properties, such as energy, heat capacity, and magnetization, are obtained from the microscopic information regarding the Hamiltonian of the system by considering the Gibbs state. This theory is summarized as equilibrium quantum statistical mechanics. Although there is no unified theory for general non-equilibrium systems, in linear response regime there is a formula for response functions to perturbations, the Kubo formula [1]. An important application of linear response theory is the electric conductivity. Being applied the electric field, the system in a stable equilibrium state is supposed to settle in another stable steady state with non-vanishing electric current (non-equilibrium steady state). In fact, the electric conductivity is discussed in [1] based on the above observation. However, from the rigorous point of view, there is a subtle point in this discussion, as explained below:

If the Kubo formula (Equation (5.12) in [1]) is naively applied to the simplest case, independently moving electrons, then the electric conductivity $\sigma$ is infinite,

$$
\sigma=\infty .
$$

The reason is understood, as follows. The formula of linear response theory is derived from the Hamiltonian dynamics of the closed system. Since the system is closed, the velocity of the electron that is accelerated by the electric field is monotonically increasing and goes to infinite in the long time limit. Thus, the electric conductivity becomes infinite finally. The issue comes from the absence of the non-equilibrium steady state (NESS) in this dynamic. In fact, a small adiabatic factor is often introduced in the practical calculation without any physical justification.

One may claim that this difficulty is due to the idealistic nature of the free electron system. In a realistic circumstance, systems are not free from their environments, and therefore the dynamics must be dissipative. The aim of this paper is to see how the Kubo formula is modified by considering the dissipative dynamics. The dissipative dynamics of a particle under electric field is studeid in [2-4]. However, these studies deal with one-particle systems and do not have NESS. We would like to 
construct a NESS in a many body system, an infinite quantum system. It is not a trivial task to construct such a model. The dissipative dynamics of many body fermion systems is discussed in [5-7]. We will use the consequence of them, especially of [5], because the physical meaning of the dynamics is clear.

This paper is organized, as follows. First, in Section 2, we explain the quantum dynamical semigroup of Davies [5] and construct a NESS. Subsequently, we derive the formula of conductivity in the lattice systems. The formula is almost the same as the Kubo formula with an adiabatic factor. However, the parameter here represents the strengthen of the dissipation. Thus, our formula can be regarded as a physical justification of the adiabatic factor. In the models that the potential is 0 $(V=0)$ the current is obtained concretely. This is the topic of Section 3. Finally, in Section 4, the conductivity of crystals (that is the system with periodic potential) is discussed. It turns out that, in the low temperature and small dissipation limit, the conductivity is given as the integral of the velocity over the Fermi surface. In Appendix A, we will treat the free continuous model and show that the Drude formula is obtained.

\section{Non-Interacting Fermion Systems and Kubo Formula}

We consider non-interacting many body systems of fermionic particles on $d$-dimensional lattice $\mathbb{Z}^{d}$. One particle is described by the Hilbert space $l^{2}\left(\mathbb{Z}^{d}\right)$ and the Hamiltonian

$$
(h \phi)(x)=-\sum_{|x-y|=1} \phi(y)+V(x) \phi(x), \phi \in l^{2}\left(\mathbb{Z}^{d}\right) .
$$

$V$ is a real valued function on $\mathbb{Z}^{d}$ called potential, and we assume $V$ is bounded. Subsequently, $h$ is a bounded self adjoint operator. For a finite subset $\Lambda_{N}=[-N, N]^{d} \cap \mathbb{Z}^{d}(N \in \mathbb{N})$, denote the corresponding projection by $P_{N}$. That is, $P_{N}: l^{2}\left(\mathbb{Z}^{d}\right) \rightarrow l^{2}\left(\mathbb{Z}^{d}\right)$ is defined as

$$
\left(P_{N} \phi\right)(x)= \begin{cases}\phi(x) & x \in \Lambda_{N} \\ 0 & \text { otherwise }\end{cases}
$$

Define $h_{N}=P_{N} h P_{N}$. Since $h_{N}$ is a finite-rank self-adjoint operator it is decomposed, as follows:

$$
h_{N}=\sum_{n=1}^{\left|\Lambda_{N}\right|} \epsilon_{n}^{(N)}\left|\phi_{n}^{(N)}\right\rangle\left\langle\phi_{n}^{(N)}\right|
$$

(here, we used the Dirac notation of physics). $\epsilon_{n}^{(N)}$ and $\phi_{n}^{(N)}$ are eigenvalues and the corresponding eigenvectors with norm 1 and orthogonal each other. When the eigenvalue is degenerated, this decomposition is not unique. However, the way of decomposition does not matter in the following discussion.

Now, we consider many particle systems on $\mathbb{Z}^{d}$. The many body system is described by the CAR algebra $\mathcal{A}^{\mathrm{CAR}}\left(l^{2}\left(\mathbb{Z}^{d}\right)\right.$ ) (we denote simply $\mathcal{A}$ below) generated by the creation/annihilation operators $a^{*}(f) / a(f)$ satisfying the following canonical anti-commutation relations:

$$
\begin{gathered}
\left\{a^{*}(f), a(g)\right\}=\langle g, f\rangle 1, \quad f, g \in l^{2}\left(\mathbb{Z}^{d}\right) \\
\{a(f), a(g)\}=0,
\end{gathered}
$$

where $\{A, B\}=A B+B A$ and $\langle\cdot, \cdot\rangle$ is the inner product of $l^{2}\left(\mathbb{Z}^{d}\right)$. Suppose that the particles are moving independently by the Hamiltonian $h$, then the automorphism of the non-interacting dynamics on the many body system $\alpha_{t}: \mathcal{A} \rightarrow \mathcal{A}$ (Heisenberg picture) is given as

$$
\alpha_{t}\left(a^{\#}\left(f_{1}\right) \cdots a^{\#}\left(f_{n}\right)\right)=a^{\#}\left(e^{i t h} f_{1}\right) \cdots a^{\#}\left(e^{i t h} f_{n}\right)
$$


where $a^{\#}$ stands for $a$ or $a^{*}$.

A state is given as a normalized positive linear functional on the algebra $\mathcal{A}$. An important class of states in the fermion systems is quasi-free state. A state $\omega$ is called a quasi-free state if the following conditions are satisfied: for any $n, m \in \mathbb{N}, f_{1}, \cdots, f_{n}, g_{1}, \cdots, g_{m} \in l^{2}\left(\mathbb{Z}^{d}\right)$,

$$
\omega\left(a^{*}\left(f_{n}\right) \cdots a^{*}\left(f_{1}\right) a\left(g_{1}\right) \cdots a\left(g_{m}\right)\right)=\delta_{n m} \operatorname{det}\left(\left(\omega\left(a^{*}\left(f_{i}\right) a\left(g_{j}\right)\right)\right)_{i j}\right) .
$$

The property of quasi-free state is completely determined by its two-point functions. Because the two-point function is always expressed by a positive operator $0 \leq R \leq 1$ on $l^{2}\left(\mathbb{Z}^{d}\right)$ as

$$
\omega\left(a^{*}(f) a(g)\right)=\langle g, R f\rangle,
$$

the quasi-free state is completely decided by an operator $R$. Conversely, given an operator $R$ on $l^{2}\left(\mathbb{Z}^{d}\right)$ that satisfies $0 \leq R \leq 1$, we have a quasi-free state by the above relations. For example, an equilibrium state $\omega_{\beta, \mu}$ (KMS state) at inverse temperature $\beta$ and chemical potential $\mu$ of a non-interacting fermion system with one-particle Hamiltonian $h$ is the quasi-free state that corresponds to an operator $f_{\beta, \mu}(h)$, where $f_{\beta, \mu}$ is the Fermi-Dirac distribution $f_{\beta, \mu}(\epsilon)=\frac{1}{1+\beta^{\beta(\epsilon-\mu)}}$.

Next, we consider dissipative dynamics on $\mathcal{A}$. We use the quantum dynamical semigroup discussed in [5].

First, let us recall the definition of the quantum dynamical semigroup in general settings. A quantum dynamical semigroup is a family of state transformations with the semigroup property.

Definition 1. Let $\mathcal{A}$ be a unital $C^{*}$-algebra. A family of linear maps on $\mathcal{A},\left\{T_{t}\right\}_{t \geq 0}$, is called a quantum dynamical semigroup if it satisfies the following conditions:

(1) $T_{t}$ is a unital $C P$ (completely positive) map for every $t \geq 0$.

(2) $T_{0}=\mathrm{id}(\mathrm{id}$ is an identity map on $\mathcal{A})$.

(3) $T_{t+s}=T_{t} \circ T_{s}(t, s \geq 0)$.

(4) $\lim _{t \downarrow 0}\left\|T_{t}(A)-A\right\|=0, \quad A \in \mathcal{A}$.

In (1), unital means the relation $T_{t}(I)=I$ and complete positivity is defined as follows; a linear map $T: \mathcal{A} \rightarrow \mathcal{A}$ is completely positive if, for any $N \in \mathbb{N}, T \otimes \mathrm{id}_{N}: \mathcal{A} \otimes M(N, \mathbb{C}) \rightarrow \mathcal{A} \otimes M(N, \mathbb{C})$ is a positive map, where $M(N, \mathbb{C})$ is the algebra of $N \times N$ complex matrices. (1) is the condition that each $T_{t}$ is a state transformation. (2) implies that at time 0 the system dose not change. (3) is the semigroup property. Here, we contain the strong continuity (4) in the definition.

Quantum dynamical semigroup is one of tools describing the dynamics of open quantum systems. This is an approximation, because, in general, the dynamics of open systems does not have the semigroup property (3). If the system is coupled to a large thermal reservoir with small relaxation time, this approximation is expected to work well. Indeed it is shown that quantum dynamical semigroup is obtained in the weak coupling limit in the physically natural settings [8].

One of the most important features of quantum dynamical semigroup is that it is determined by its generator $L$, defined as

$$
L(A)=\lim _{t \downarrow 0} \frac{T_{t}(A)-A}{t} .
$$

This is defined only for $A \in \mathcal{A}$, such that the limit (the norm limit) of the right hand side exits. The set of such elements $\mathcal{D}(L)$ (the domain of $L$ ) forms a dense subspace of $\mathcal{A}$.

Now, we turn to the fermion systems on the lattice $\mathbb{Z}^{d}$ and introduce a model. Here, we want to deal with the system coupled to a heat reservoir. The dynamics must drive the states to an equilibrium state determined by the reservoir. As the system itself is an infinite system, it is not a trivial task to construct such a model. One of the possible models was given by Davies. Davies considered, in [5], the dissipative dynamics on many body fermion systems, which is described by the language 
of one-particle Hilbert space. Let $\delta$ be the generator of the non-interacting dynamics $\alpha_{t}$. Define a *automorphism $\theta: \mathcal{A} \rightarrow \mathcal{A}$ by $\theta\left(a^{\#}(f)\right)=-a^{\#}(f)$. For each $N \in \mathbb{N}$, define a linear map $L_{N}: \mathcal{A} \rightarrow \mathcal{A}$ by $\mathcal{D}\left(L_{N}\right)=\mathcal{D}(\delta)$ and

$$
\begin{aligned}
L_{N}(A) \quad & =\delta(A) \\
& +\lambda \sum_{n=1}^{\left|\Lambda_{N}\right|} f_{\beta, \mu}\left(\epsilon_{n}^{(N)}\right)\left(2 a\left(\phi_{n}^{(N)}\right) \theta(A) a^{*}\left(\phi_{n}^{(N)}\right)-a\left(\phi_{n}^{(N)}\right) a^{*}\left(\phi_{n}^{(N)}\right) A-A a\left(\phi_{n}^{(N)}\right) a^{*}\left(\phi_{n}^{(N)}\right)\right) \\
& +\lambda \sum_{n=1}^{\left|\Lambda_{N}\right|}\left(1-f_{\beta, \mu}\left(\epsilon_{n}^{(N)}\right)\right)\left(2 a^{*}\left(\phi_{n}^{(N)}\right) \theta(A) a\left(\phi_{n}^{(N)}\right)-a^{*}\left(\phi_{n}^{(N)}\right) a\left(\phi_{n}^{(N)}\right) A-A a^{*}\left(\phi_{n}^{(N)}\right) a\left(\phi_{n}^{(N)}\right)\right)
\end{aligned}
$$

for $A \in \mathcal{D}\left(L_{N}\right) . \lambda$ is a positive real number representing the strengthen of dissipation. The first term of the right hand side corresponds to the Hamiltonian dynamics, the second term represents the creation of particles in the eigenstate of energy by the distribution $f_{\beta, \mu}$, and the third term means the annihilation of particles by the distribution $\left(1-f_{\beta, \mu}\right)$.

This map $L_{N}$ generates a quantum dynamical semigroup, we denote it $\left\{T_{t}^{N}\right\}_{t \geq 0}$. The limit $\lim _{N \rightarrow \infty} T_{t}^{N}(A)$ exists for each $t \geq 0, A \in \mathcal{A}$ and define a quantum dynamical semigroup $T_{t}(A)=$ $\lim _{N \rightarrow \infty} T_{t}^{N}(A)$.

It is shown that every state $\psi$ converges to $\omega_{\beta, \mu}$, the equilibrium state at inverse temperature $\beta$ and chemical potential $\mu$, in the weak* topology by the dynamics $T_{t}$ :

$$
\lim _{t \rightarrow \infty} \psi \circ T_{t}(A)=\omega_{\beta, \mu}(A), \quad A \in \mathcal{A} \text {. }
$$

Thus, $T_{t}$ is considered to describe the dynamics of the system coupled to a thermal reservoir. For the detail of this dynamics, see [5].

Now, suppose that the system is initially in the stable equilibrium state $\omega_{\beta, \mu}$ under the dynamics $T_{t}$ and at time $t=0$ applied the uniform electric field $E$ in the direction $e_{1}=(1,0, \cdots, 0) \in \mathbb{Z}^{d}$. SUbsequently, one-particle Hamiltonian is changed to $h_{E}=h-E Q_{1}$, where $Q_{1}$ is the position operator; the domain is $\mathcal{D}\left(Q_{1}\right)=\left\{\left.\phi \in l^{2}\left(\mathbb{Z}^{d}\right)\left|\sum_{x \in \mathbb{Z}^{d}}\right| x_{1}\right|^{2}|\phi(x)|^{2}\right\}$ and, for $\phi \in \mathcal{D}\left(Q_{1}\right), Q_{1}$, acts as

$$
\left(Q_{1} \phi\right)(x)=x_{1} \phi(x)
$$

where $x_{1}$ is the first element of $x \in \mathbb{Z}^{d} . Q_{1}$ is an unbounded self adjoint operator. Thus, $h_{E}$ is also an unbounded self adjoint operator with the domain $\mathcal{D}\left(h_{E}\right)=\mathcal{D}\left(Q_{1}\right)$. Let $\delta_{E}$ be the generator of the non-interacting dynamics on $\mathcal{A}$ with the one-particle Hamiltonian $h_{E}$, and define a quantum dynamical semigroup $T_{t}^{E, N}$ by replacing $\delta$ in the definition of $L_{N}$ to $\delta_{E}$. These maps also define a quantum dynamical semigroup $T_{t}^{E}$ in the limit $N \rightarrow \infty$. This dynamics maps a quasi-free state to another quasi-free state. Let $\phi$ be a quasi-free state with the operator $R$, then $\phi \circ T_{t}^{E}$ is also a quasi-free state and the corresponding operator is

$$
e^{-2 \lambda t} e^{-i t h_{E}} R e^{i t h_{E}}+2 \lambda \int_{0}^{t} e^{-2 \lambda s} e^{-i s h_{E}} f_{\beta, \mu}(h) e^{i s h_{E}} d s
$$

Thus, the system initially in the equilibrium state $\omega_{\beta, \mu}$ finally converges to the stable non-equilibrium steady state by the dynamics $T_{t}^{E}$ in the weak* topology (in fact the limit does not depend on the initial state). We denote this state by $\omega_{\beta, \mu}^{\lambda, E}$ (apart from the case that $E=0$; this limit depends on $\lambda$ ). $\omega_{\beta, \mu}^{\lambda, E}$ is a quasi-free state corresponding to the operator

$$
2 \lambda \int_{0}^{\infty} e^{-2 \lambda s} e^{-i s h_{E}} f_{\beta, \mu}(h) e^{i s h_{E}} d s
$$


Now, let us consider the electric current. The increase of the number of particles at site $x \in \mathbb{Z}^{d}$ per unit time is expressed as

$$
\delta\left(a_{x}^{*} a_{x}\right)=\sum_{|x-y|=1} i\left(a_{x}^{*} a_{y}-a_{y}^{*} a_{x}\right) .
$$

Here, we write simply $a^{\#}\left(\eta_{x}\right)$ as $a_{x}^{\#}$, where $\left\{\eta_{x}\right\}_{x \in \mathbb{Z}^{d}}$ is the standard basis of $l^{2}\left(\mathbb{Z}^{d}\right): \eta_{x}(y)=\delta_{x y}$. Each term in the summation represents the number of particles flowing from the nearest neighbor site of $x \in \mathbb{Z}^{d}$ per unit time. Define the observable $\hat{j}_{1, x}$ representing the current at $x$ in the direction $e_{1}$ as the average of the current flowing form $x-e_{1}$ to $x$ and the current from $x$ to $x+e_{1}$;

$$
\hat{j}_{1, x}=\frac{i}{2}\left(a_{x}^{*} a_{x+e_{1}}-a_{x+e_{1}}^{*} a_{x}+a_{x-e_{1}}^{*} a_{x}-a_{x}^{*} a_{x+e_{1}}\right)
$$

The current in the non-equilibrium steady state $\omega_{\beta, \mu}^{\lambda, E}$ is

$$
j_{1, x}^{\lambda}(E ; \beta, \mu) \equiv \omega_{\beta, \mu}^{\lambda, E}\left(\hat{j}_{1, x}\right)
$$

The following theorem, one of our main results, shows that the response of the current $j_{1, x}^{\lambda}(E ; \beta, \mu)$ to the electric field $E$ is simply expressed by the formula while using the information of the one-particle Hamiltonian.

Theorem 1. The current $j_{1, x}^{\lambda}(E ; \beta, \mu)$ is differentiable at $E=0$ and its derivative $\sigma_{1, x}^{\lambda}(\beta, \mu)$ (electric conductivity) is expressed as

$$
\sigma_{1, x}^{\lambda}(\beta, \mu)=\operatorname{Re} \int_{0}^{\infty} e^{-2 \lambda s}\left\langle\eta_{x}, e^{-i s h} i\left[Q_{1}, f_{\beta, \mu}(h)\right] e^{i s h} \eta_{1} \eta_{x}\right\rangle d s,
$$

where $\operatorname{Re}$ is the real part and $v_{1}=i\left[h, Q_{1}\right]$ is the velocity ( $v_{1}$ is independent of the potential $V$ ).

Proof. First, let us calculate the two point functions $\omega_{\beta, \mu}^{\lambda, E}\left(a_{x}^{*} a_{y}\right)$.

$$
\omega_{\beta, \mu}^{\lambda, E}\left(a_{x}^{*} a_{y}\right)=2 \lambda \int_{0}^{\infty} e^{-2 \lambda s}\left\langle e^{i s h_{E}} \eta_{y}, f_{\beta, \mu}(h) e^{i s h_{E}} \eta_{x}\right\rangle d s
$$

Because $\eta_{x}, \eta_{y} \in \mathcal{D}\left(Q_{1}\right)=\mathcal{D}\left(h_{E}\right),\left\langle e^{i s h_{E}} \eta_{y}, f_{\beta, \mu}(h) e^{i s h_{E}} \eta_{x}\right\rangle$ is differentiable by $s$. Integrating by parts we obtain

$$
\begin{aligned}
\omega_{\beta, \mu}^{\lambda, E}\left(a_{x}^{*} a_{y}\right)= & \left\langle\eta_{y}, f_{\beta, \mu}(h) \eta_{x}\right\rangle \\
& +\int_{0}^{\infty} e^{-2 \lambda s}\left(\left\langle i h_{E} e^{i s h_{E}} \eta_{y}, f_{\beta, \mu}(h) e^{i s h_{E}} \eta_{x}\right\rangle+\left\langle e^{i s h_{E}} \eta_{y}, f_{\beta, \mu}(h) i h_{E} e^{i s h_{E}} \eta_{x}\right\rangle\right) d s \\
= & \omega_{\beta, \mu}\left(a_{x}^{*} a_{y}\right) \\
& +i E \int_{0}^{\infty} e^{-2 \lambda s}\left(\left\langle Q_{1} e^{i s h_{E}} \eta_{y}, f_{\beta, \mu}(h) e^{i s h_{E}} \eta_{x}\right\rangle-\left\langle e^{i s h_{E}} \eta_{y}, f_{\beta, \mu}(h) Q_{1} e^{i s h_{E}} \eta_{x}\right\rangle\right) d s
\end{aligned}
$$

We have $e^{i s h} \eta_{z} \in \mathcal{D}\left(Q_{1}\right)\left(z \in \mathbb{Z}^{d}\right)$ and, for any $\phi \in l^{2}\left(\mathbb{Z}^{d}\right), \lim _{E \rightarrow 0} e^{i s h_{E}} \phi=e^{i s h} \phi$. In addition, because $\left|\left\langle\eta_{x}, f_{\beta, \mu}(h) \eta_{y}\right\rangle\right|$ decays exponentially with respect to $|x-y|$ [9], for any $\phi \in \mathcal{D}\left(Q_{1}\right), f_{\beta, \mu}(h) \phi \in \mathcal{D}\left(Q_{1}\right)$ and $\left[Q_{1}, f_{\beta, \mu}(h)\right] \equiv Q_{1} f_{\beta, \mu}(h)-f_{\beta, \mu}(h) Q_{1}$ can be extended to a bounded operator. Thus,

$$
\lim _{E \rightarrow 0}\left\langle\eta_{y}, e^{-i s h_{E}}\left[Q_{1}, f_{\beta, \mu}(h)\right] e^{i s h_{E}} \eta_{x}\right\rangle=\left\langle\eta_{y}, e^{-i s h}\left[Q_{1}, f_{\beta, \mu}(h)\right] e^{i s h} \eta_{x}\right\rangle .
$$


In addition, one can exchange the limit and integral and obtain

$$
\lim _{E \rightarrow 0} \frac{\omega_{\beta, \mu}^{\lambda, E}\left(a_{x}^{*} a_{y}\right)-\omega_{\beta, \mu}\left(a_{x}^{*} a_{y}\right)}{E}=\int_{0}^{\infty} e^{-2 \lambda s}\left\langle\eta_{y}, e^{-i s h} i\left[Q_{1}, f_{\beta, \mu}(h)\right] e^{i s h} \eta_{x}\right\rangle d s .
$$

The relation

$$
v_{l} \eta_{z}=i\left[h, Q_{1}\right] \eta_{z}=i \eta_{z+e_{1}}-i \eta_{z-e_{1}}
$$

and the definition of $\hat{j}_{1, x}$ concludes the formula.

Here, we would like to mention the relation between the original Kubo formula and our formula. According to the paper [1], when the perturbation $f A(|f| \ll 1)$ is added to the Hamiltonian $H$, the change of the first order $\triangle B$ of quantity $B$ in NESS is

$$
\Delta B=\frac{1}{i} \int_{0}^{\infty} \operatorname{Tr}\left[A, \rho_{\beta, \mu}\right] B(t) d t=i \int_{0}^{\infty} \operatorname{Tr} \rho_{\beta, \mu}[A, B(t)] d t
$$

where $B(t)=e^{i t H} B e^{-i t H}$ and $\rho_{\beta, \mu}$ is the Gibbs state. Or, adding the adiabatic factor $e^{-\epsilon t}$ to converges the integral,

$$
\Delta B=\lim _{\epsilon \downarrow 0} i \int_{0}^{\infty} e^{-\epsilon t} \operatorname{Tr} \rho_{\beta, \mu}[A, B(t)] d t .
$$

However, as discussed in the introduction, even though the adiabatic factor is added, if one takes the limit $\epsilon \downarrow 0, \Delta B$ may diverge (for example the electric current in the free model). Additionally, the physical meaning of the parameter $\epsilon$ is not clear. Our formula is changed to

$$
i \int_{0}^{\infty} e^{-2 \lambda t} \omega_{\beta, \mu}\left(\left[\sum_{n \in \mathbb{Z}} n a_{n}^{*} a_{n}, \hat{j}_{x}(t)\right]\right) d t
$$

where $\hat{j}_{x}(t)=e^{i t H} \hat{j}_{x} e^{-i t H}$ ( $H$ is the total Hamiltonian without perturbation). This is same as the Kubo formula with the adiabatic factor (not taking the limit $\lambda \downarrow 0$ ) in the case $A=\sum_{n \in \mathbb{Z}} n a_{n}^{*} a_{n}, B=\hat{j}_{x}$. The difference between the original Kubo formula and our approach is summarized, as follows: original

- considering the Hamiltonian dynamics of closed system;

- NESS and the convergence to it are not discussed; and,

- the adiabatic factor $e^{-\epsilon t}$ is artificial and the physical meaning of the parameter $\epsilon$ is not clear;

ours

- considering the dissipative dynamics and discussing the convergence to NESS; and,

- the factor $e^{-2 \lambda t}$ emerges naturally from the dissipative model and the physical meaning of the parameter $\lambda$ is clear, the strengthening of the dissipation or the inverse of relaxation time.

Although, in this paper, we do not discuss the magnetic field, the formula can be extended easily to the case that the magnetic field is present and it produces the TKNN formula [10,11] in the limit $\beta \rightarrow \infty, \lambda \downarrow 0$.

\section{Solvable Model}

In the previous section, we derived the formula of electric conductivity for the general form of potential $V$. In fact, in the case where $V=0$, one can calculate the current explicitly. Here for simplicity we restrict the discussion to one-dimensional systems. Which is, the one-particle Hilbert space is $l^{2}(\mathbb{Z})$. The one-particle Hamiltonian for potential $V=0$ is the multiplication operator on the momentum space $L^{2}(-\pi, \pi)$. Precisely the one-particle Hamiltonian is given by the multiplication operator on $L^{2}(-\pi, \pi)$

$$
(\hat{h} \phi)(k)=(-\cos k) \phi(k), \phi \in L^{2}(-\pi, \pi)
$$


and the Fourier transformation $\mathcal{F}: l^{2}(\mathbb{Z}) \rightarrow L^{2}(-\pi, \pi)$ by $h=\mathcal{F}^{-1} \hat{h} \mathcal{F}$. As discussed in Section 2, the system finally converges to a unique steady quasi-free state with the operator

$$
R_{\beta, \mu}^{\lambda, E} \equiv 2 \lambda \int_{0}^{\infty} e^{-2 \lambda s} e^{-i s h_{E}} f_{\beta, \mu}(h) e^{i s h_{E}} d s .
$$

Here, we will obtain the explicit form of $R_{\beta, \mu}^{\lambda, E}$. In the following, we consider in the momentum space and identify $\mathcal{F}^{-1} h \mathcal{F}$ as $h$. Note that $Q$ is a differential operator on the momentum spaces, thus

$$
\begin{aligned}
& \left(e^{i s h} \phi\right)(k)=e^{i \epsilon(k) s} \phi(k) \\
& \left(e^{i s Q} \phi\right)(k)=\phi(k+s),
\end{aligned}
$$

where $\epsilon(k)=-\cos k$. Using the product formula [12]

$$
e^{i s h_{E}} \phi=\lim _{n \rightarrow \infty}\left(e^{i \frac{s}{n} h} e^{-i \frac{s}{n} E Q}\right)^{n} \phi
$$

we obtain

$$
\left(e^{i s h_{E}} \phi\right)(k)=\exp \left(i s \int_{0}^{1} \epsilon(k-E s \xi) d \xi\right) \phi(k-E s),
$$

and

$$
\begin{aligned}
\left\langle\psi, R_{\beta, \mu}^{\lambda, E} \phi\right\rangle & =2 \lambda \int d k \int_{0}^{\infty} d s e^{-2 \lambda s} \overline{\psi(k-s E)} f_{\beta, \mu}(\epsilon(k)) \phi(k-s E) \\
& =2 \lambda \int_{0}^{\infty} d s \int d k \overline{\psi(k)} e^{-2 \lambda s} f_{\beta, \mu}(\epsilon(k+s E)) \phi(k) \\
& =\int d k \overline{\psi(k)}\left(2 \lambda \int_{0}^{\infty} e^{-2 \lambda s} f_{\beta, \mu}(\epsilon(k+s E)) d s\right) \phi(k) d k .
\end{aligned}
$$

Thus, $R_{\beta, \mu}^{\lambda, E}$ is the multiplication operator of the function

$$
\left(R_{\beta, \mu}^{\lambda, E}\right)(k)=2 \lambda \int_{0}^{\infty} e^{-2 \lambda s} f_{\beta, \mu}(\epsilon(k+s E)) d s .
$$

Now, let us calculate the current. Note that it is independent of the site (so we denote the current by $\left.j^{\lambda}(E ; \beta, \mu)\right)$ and corresponds to the integration of the velocity $\frac{d}{d k} \epsilon(k)=-\sin k$ :

$$
\begin{aligned}
j^{\lambda}(E ; \beta, \mu) & =\frac{1}{2 \pi} \int_{-\pi}^{\pi}(-\sin k)\left(2 \lambda \int_{0}^{\infty} e^{-2 \lambda s} f_{\beta, \mu}(\epsilon(k+s E)) d s\right) d k \\
& =\frac{1}{2 \pi} \int_{-\pi}^{\pi}\left(2 \lambda \int_{0}^{\infty}(-\sin (k-s E)) e^{-2 \lambda s} d s\right) f_{\beta, \mu}(\epsilon(k)) d k \\
& =\frac{2 \lambda E}{4 \lambda^{2}+E^{2}} \int_{-\pi}^{\pi} \frac{-\cos k}{1+e^{-\beta(\cos k+\mu)}} \frac{d k}{2 \pi} .
\end{aligned}
$$

The current becomes 0 in the limit $E \rightarrow 0$ and $\lambda \downarrow 0$, respectively. On the other hand, the conductivity is

$$
\sigma_{l}^{\lambda}(\beta, \mu)=\frac{1}{2 \lambda} \int_{-\pi}^{\pi} \frac{-\cos k}{1+e^{\beta(-\cos k-\mu)}} \frac{d k}{2 \pi}
$$

and it goes to infinite as $\lambda \downarrow 0$. 


\section{Electric Conductivity in Crystals}

In this section, we consider the electrons in crystals, which is the electrons under periodic potentials. Suppose that the potential $V$ has period $p_{l} \in \mathbb{N}$ in the direction $e_{l}(l=1,2, \cdots, d)$;

$$
V\left(x+p_{1} e_{1}\right)=V\left(x+p_{2} e_{2}\right)=\cdots=V\left(x+p_{d} e_{d}\right)=V(x), x \in \mathbb{Z}^{d} .
$$

Take $\Lambda=\left\{m_{1} e_{1}+\cdots m_{d} e_{d} \mid 0 \leq m_{1}<p_{1}, \cdots 0 \leq m_{d}<p_{d}\right\}$ and $\mathcal{B}=\mathbb{R}^{d} /\left(p_{1} \mathbb{Z} \times \cdots \times p_{d} \mathbb{Z}\right)=$ $\left(-\frac{\pi}{p_{1}}, \frac{\pi}{p_{1}}\right] \times \cdots\left(-\frac{\pi}{p_{d}}, \frac{\pi}{p_{d}}\right] \cdot \mathcal{B}$ is called the Brillouin zone. For periodic potentials, we can use the Bloch theory. The Hilbert space $l^{2}\left(\mathbb{Z}^{d}\right)$ is decomposed as the following direct integral

$$
l^{2}\left(\mathbb{Z}^{d}\right)=\int_{\mathcal{B}}^{\oplus} \mathcal{H}_{k} d k
$$

where $\mathcal{H}_{k}=l^{2}(\Lambda) \simeq \mathbb{C}^{|\Lambda|}$. Additionally, by the periodicity of $h$, this is decomposed as

$$
h=\int_{\mathcal{B}}^{\oplus} h_{k} d k,
$$

where $h_{k}$ is an operator on $l^{2}(\Lambda)$ defined as

$$
\left(h_{k} \phi\right)(x)=-\sum_{|x-y|=1} \phi(y)+V(x) \phi(x), \quad x \in \Lambda
$$

with boundary conditions $\phi\left(x+p_{j} e_{j}\right)=e^{i k p_{j}} \phi(x)$. By this decomposition, we also have

$$
f_{\beta, \mu}(h)=\int_{\mathcal{B}}^{\oplus} f_{\beta, \mu}\left(h_{k}\right) d k
$$

Because the commutator with the position operator $Q_{1}$ has the same periodicity, it is decomposed and given by the derivative:

$$
i\left[Q_{1}, f_{\beta, \mu}(h)\right]=\int_{\mathcal{B}}^{\oplus} \partial_{k_{1}} f_{\beta, \mu}\left(h_{k}\right) d k
$$

The velocity $v_{1}$ is also decomposed as

$$
v_{1}=i\left[h, Q_{1}\right]=\int_{\mathcal{B}}^{\oplus} v_{1, k} d k,
$$

where $v_{1, k}=-\partial_{k_{1}} h_{k}$.

Consider the mean of the conductivity

$$
\sigma_{1}^{\lambda}(\beta, \mu)=\frac{1}{|\Lambda|} \sum_{x \in \Lambda} \sigma_{1, x}^{\lambda}(\beta, \mu)
$$

then this is expressed as

$$
\sigma_{1}^{\lambda}(\beta, \mu)=\operatorname{Re} \int_{0}^{\infty} e^{-2 \lambda s}\left(\int_{\mathcal{B}} \operatorname{Tr} e^{-i s h_{k}} \partial_{k_{1}} f_{\beta, \mu}\left(h_{k}\right) e^{i s h_{k}} v_{1, k} d k\right) d s
$$

In the following, we consider the low temperature and small dissipation regime. Here, we impose some assumptions.

Let $\epsilon_{k}^{n}$ and $\psi_{k}^{n}$ be eigenvalues and eigenvectors of $h_{k}(n=1,2, \cdots,|\Lambda|)$. Suppose

- $\quad h_{k}$ is nondegenerate for all $k \in \mathcal{B}$

- the eigenvalues $\epsilon_{k}^{n}$ and eigenvectors $\psi_{k}^{n}$ of $h_{k}$ are in $C^{2}$-class. 
Theorem 2. Under the above assumptions, we have

$$
\sigma_{1}^{\lambda}(\beta, \mu)=\frac{1}{2 \lambda} \sum_{n=1}^{|\Lambda|} \int_{\mathcal{B}} f_{\beta, \mu}\left(\epsilon_{k}^{n}\right) \partial_{k_{1}}^{2} \epsilon_{k}^{n} d k+O(\lambda) .
$$

Especially in the low temperature limit $\beta \rightarrow \infty$, electric conductivity $\sigma_{1}^{\lambda}(\infty, \mu)$ is expressed as

$$
\begin{aligned}
\sigma_{1}^{\lambda}(\infty, \mu) & =\frac{1}{2 \lambda} \sum_{n=1}^{|\Lambda|} \int_{\partial \mathcal{B}_{\mu}^{n}}\left\langle\psi_{k}^{n}, v_{1, k} \psi_{k}^{n}\right\rangle n_{1}(k) d k+O(\lambda) \\
& =\frac{1}{2 \lambda} \sum_{n=1}^{|\Lambda|} \int_{\partial \mathcal{B}_{\mu}^{n}} \partial_{k_{1}} \epsilon_{k}^{n} n_{1}(k) d k+O(\lambda)
\end{aligned}
$$

where $\mathcal{B}_{\mu}^{n}=\left\{k \in \mathcal{B} \mid \epsilon_{k}^{n} \leq \mu\right\}$ and $\partial \mathcal{B}_{\mu}^{n}$ is the boundary of $\mathcal{B}_{\mu}^{n}$ and $n_{l}(k)$ is the l-th element of the unit normal vector at $k \in \partial \mathcal{B}_{\mu}^{n}$. If $\mu$ is in the band gap, $\mathcal{B}_{\mu}^{n}$ has no boundary and the above integral is 0 .

This formula means that the main contribution to the conductivity in low temperature regime is given by integral of the velocity over the Fermi surface. Additionally, if $\mu$ is in the band gap, the conductivity is almost 0 (insulator).

Proof. Because Tre $e^{-i s h_{k}} \partial_{k_{1}} f_{\beta, \mu}\left(h_{k}\right) e^{i s h_{k}} \partial_{k_{1}} h_{k}$ is bounded for $k$, by the Fubini theorem, we have

$$
\sigma_{1}^{\lambda}(\beta, \mu)=\operatorname{Re} \int_{\mathcal{B}}\left(\int_{0}^{\infty} e^{-2 \lambda s} \operatorname{Tr} e^{-i s h_{k}} \partial_{k_{1}} f_{\beta, \mu}\left(h_{k}\right) e^{i s h_{k}} \partial_{k_{1}} h_{k} d s\right) d k
$$

Put $P_{k}^{n}=\left|\psi_{k}^{n}\right\rangle\left\langle\psi_{k}^{n}\right|$. As $h_{k}$ is nondegenerate, we get

$$
\begin{aligned}
& \int_{0}^{\infty} e^{-2 \lambda s} \operatorname{Tr} e^{-i s h_{k}} \partial_{k_{1}} f_{\beta, \mu}\left(h_{k}\right) e^{i s h_{k}} \partial_{k_{1}} h_{k} d s \\
= & \sum_{n=1}^{|\Lambda|} \sum_{m=1}^{|\Lambda|} \int_{0}^{\infty} e^{-\left(2 \lambda+i \epsilon_{k}^{n}-i \epsilon_{k}^{m}\right) s} d s \operatorname{Tr} P_{k}^{n} \partial_{k_{1}} f_{\beta, \mu}\left(h_{k}\right) P_{k}^{m} \partial_{k_{1}} h_{k} \\
= & \frac{1}{2 \lambda} \sum_{n=1}^{|\Lambda|} \operatorname{Tr} P_{k}^{n} \partial_{k_{1}} f_{\beta, \mu}\left(h_{k}\right) P_{k}^{n} \partial_{k_{1}} h_{k} \\
& +\sum_{n \neq m} \frac{1}{2 \lambda+i\left(\epsilon_{k}^{n}-\epsilon_{k}^{m}\right)} \operatorname{Tr} P_{k}^{n} \partial_{k_{1}} f_{\beta, \mu}\left(h_{k}\right) P_{k}^{m} \partial_{k_{1}} h_{k} .
\end{aligned}
$$

We estimate the right hand side using the following equations. In the sequel, we skip the index $k$ and write $\partial_{k_{1}}, \epsilon_{k}^{n}$ as $\partial, \epsilon^{n}$ for short.

By differentiating $P^{n}=P^{n} P^{n}$, we have $\partial P^{n}=\left(\partial P^{n}\right) P^{n}+P^{n} \partial P^{n}$. Multiplying $P^{n}$ from both side, $P^{n}\left(\partial P^{n}\right) P^{n}=0$. Since $P^{n} P^{m}=0$ for $n \neq m$, we have $\left(\partial P^{n}\right) P^{m}+P^{n} \partial P^{m}=0$. Multiplying $P^{n}$ from both side, $P^{n}\left(\partial P^{m}\right) P^{n}=0$. From these equations, we obtain

$$
\sum_{n=1}^{|\Lambda|} \operatorname{Tr} P^{n} \partial f_{\beta, \mu}(h) P^{n} \partial h=\sum_{n=1}^{|\Lambda|} \partial f_{\beta, \mu}\left(\epsilon^{n}\right) \partial \epsilon^{n} .
$$

Additionally, if $n \neq m$, we have

$$
\begin{aligned}
P^{n} \partial f_{\beta, \mu}(h) P^{m} & =\sum_{l=1}^{|\Lambda|} f_{\beta, \mu}\left(\epsilon^{l}\right) P^{n}\left(\partial P^{l}\right) P^{m} \\
& =f_{\beta, \mu}\left(\epsilon^{m}\right) P^{n}\left(\partial P^{m}\right) P^{m}+f_{\beta, \mu}\left(\epsilon^{n}\right) P^{n}\left(\partial P^{n}\right) P^{m} \\
& =f_{\beta, \mu}\left(\epsilon^{m}\right) P^{n} \partial P^{m}+f_{\beta, \mu}\left(\epsilon^{n}\right)\left(\partial P^{n}\right) P^{m} .
\end{aligned}
$$


In addition, we obtain $P^{m} \partial h P^{n}=\epsilon^{m}\left(\partial P^{m}\right) P^{n}+\epsilon^{n} P^{m} \partial P^{n}$ similarly. Therefore, we have

$$
\begin{aligned}
\operatorname{Tr} P^{n} \partial f_{\beta, \mu}(h) P^{m} \partial h= & f_{\beta, \mu}\left(\epsilon^{m}\right) \epsilon^{m} \operatorname{Tr} P^{n}\left(\partial P^{m}\right)^{2}+f_{\beta, \mu}\left(\epsilon^{m}\right) \epsilon^{n} \operatorname{Tr} P^{n}\left(\partial P^{m}\right) P^{m} \partial P^{n} \\
& +f_{\beta, \mu}\left(\epsilon^{n}\right) \epsilon^{m} \operatorname{Tr}\left(\partial P^{n}\right) P^{m}\left(\partial P^{m}\right) P^{n}+f_{\beta, \mu}\left(\epsilon^{n}\right) \epsilon^{n} \operatorname{Tr} P^{m}\left(\partial P^{n}\right)^{2} \\
= & f_{\beta, \mu}\left(\epsilon^{m}\right)\left(\epsilon^{m}-\epsilon^{n}\right) \operatorname{Tr} P^{n}\left(\partial P^{m}\right)^{2}+f_{\beta, \mu}\left(\epsilon^{n}\right)\left(\epsilon^{n}-\epsilon^{m}\right) \operatorname{Tr} P^{m}\left(\partial P^{n}\right)^{2} .
\end{aligned}
$$

Using the above equations

$$
\sum_{n \neq m} \frac{1}{2 \lambda+i\left(\epsilon_{k}^{n}-\epsilon_{k}^{m}\right)} \operatorname{Tr}_{k}^{n} \partial_{k_{1}} f_{\beta, \mu}\left(h_{k}\right) P_{k}^{m} \partial_{k_{1}} h_{k}=\sum_{n \neq m} \frac{4 \lambda\left(\epsilon_{k}^{n}-\epsilon_{k}^{m}\right)}{4 \lambda^{2}+\left(\epsilon_{k}^{m}-\epsilon_{k}^{n}\right)^{2}} f_{\beta, \mu}\left(\epsilon_{k}^{m}\right) \operatorname{Tr} P_{k}^{n}\left(\partial_{k_{1}} P_{k}^{m}\right)^{2}
$$

Because $\epsilon_{k}^{n}$ is continuous for $k$ and $\epsilon_{k}^{n} \neq \epsilon_{k}^{m}(n \neq m)$, there is a positive constant $C$, such that, for all $k \in \mathcal{B}$ and $n \neq m,\left|\epsilon_{k}^{n}-\epsilon_{k}^{m}\right|>C$. Therefore, we obtain the $\mu$-independent upper bound

$$
\begin{gathered}
\left|\operatorname{Re} \int_{\mathcal{B}} \sum_{n \neq m} \frac{1}{2 \lambda+i\left(\epsilon_{k}^{n}-\epsilon_{k}^{m}\right)} \operatorname{Tr} P_{k}^{n} \partial_{k_{1}} f_{\beta, \mu}\left(h_{k}\right) P_{k}^{m} \partial_{k_{1}} h_{k}\right| \leq \frac{4 \lambda}{C} \sum_{n \neq m} \int_{\mathcal{B}} \operatorname{Tr} P_{k}^{n}\left(\partial_{k_{1}} P_{k}^{m}\right)^{2} d k . \\
\sigma_{1}^{\lambda}(\beta, \mu)=-\frac{1}{2 \lambda} \sum_{n=1}^{|\Lambda|} \int_{\mathcal{B}} \partial_{k_{1}} f_{\beta, \mu}\left(\epsilon_{k}^{n}\right) \partial_{k_{1}} \epsilon_{k}^{n} d k+O(\lambda)=\frac{1}{2 \lambda} \sum_{n=1}^{|\Lambda|} \int_{\mathcal{B}} f_{\beta, \mu}\left(\epsilon_{k}^{n}\right) \partial_{k_{1}}^{2} \epsilon_{k}^{n} d k+O(\lambda) .
\end{gathered}
$$

Put $\mathcal{B}_{\mu}^{n}=\left\{k \in \mathcal{B} \mid \epsilon_{k}^{n} \leq \mu\right\}$, then

$$
\begin{aligned}
\sigma_{1}^{\lambda}(\infty, \mu)=\lim _{\beta \rightarrow \infty} \sigma_{1}^{\lambda}(\beta, \mu) & =\frac{1}{2 \lambda} \sum_{n=1}^{|\Lambda|} \int_{\mathcal{B}_{\mu}^{n}} \partial_{k_{1}}^{2} \epsilon_{k}^{n} d k+O(\lambda) \\
& =\frac{1}{2 \lambda} \sum_{n=1}^{|\Lambda|} \int_{\partial \mathcal{B}_{\mu}^{n}} \partial_{k_{1}} \epsilon_{k}^{n} n_{1}(k) d k+O(\lambda)
\end{aligned}
$$

In one-dimensional case $(d=1)$, the absence of degeneracy of eigenvalues $\epsilon_{k}^{n}$ implies the fact that all of the gaps are open. Thus, $\mu$ is either in the gap or in the only one band ( $n$-th band). Furthermore $\epsilon_{k}^{n}$ is an even function and monotonically increasing or decreasing on $0 \leq k \leq \frac{\pi}{p}$. Denote $k_{\mu} \in\left[0, \frac{\pi}{p}\right]$ the solution of $\epsilon_{k}^{n}=\mu$. The conductivity is, as follows, in each case

$$
\begin{gathered}
\sigma^{\lambda}(\infty, \mu)=\left.\frac{1}{\lambda} \partial_{k} \epsilon_{k}^{n}\right|_{k=k_{\mu}}+O(\lambda)\left(\epsilon_{k}^{n} \text { is increasing on } 0 \leq k \leq \frac{\pi}{p}\right) \\
\sigma^{\lambda}(\infty, \mu)=-\left.\frac{1}{\lambda} \partial_{k} \epsilon_{k}^{n}\right|_{k=k_{\mu}}+O(\lambda)\left(\epsilon_{k}^{n} \text { is decreasing on } 0 \leq k \leq \frac{\pi}{p}\right) .
\end{gathered}
$$

\section{Discussion}

In this paper, we considered the conductivity of non-interacting lattice fermion system coupled to a heat reservoir. We dealt with this open system by the quantum dynamical semigroup discussed by Davies and constructed a NESS. The main result is the formula in Theorem 2.1 and the justification of an adiabatic factor, $e^{-2 \lambda t}$, of the Kubo formula. In our approach, the parameter $\lambda$ is naturally emerged by considering the dissipative dynamics and has the physical meaning, the strengthening of the dissipation, or the inverse of relaxation time.

In the case of periodic potentials, we showed that the conductivity is given by the integral of the velocity over the Fermi surface in the low temperature and small dissipation limit. 
Non-equilibrium statistical mechanics is a frontier of physics. Especially, NESS with a non-vanishing current is one of main themes of it [13-17]. However, there are a few concrete models [18,19]. Our derivation of the Kubo formula provides a new example of NESS.

Justifying our model from more realistic setting is our future work.

Funding: This research received no external funding.

Conflicts of Interest: The author declares no conflict of interest.

\section{Appendix A. Continuous Model and Drude Formula}

In this paper we discussed lattice models. One can also consider the dissipative dynamics introduced in Section 2 on continuous models. In this appendix we discuss the free continuous model and show that Drude formula is obtained from this model.

For simplicity, here we consider a one-dimensional system as in Section 3. In free continuous model, the one-particle Hilbert space is $L^{2}(\mathbb{R})$ and the one-particle Hamiltonian is the Fourier transformation of the multiplication operator:

$$
(\hat{h} \phi)(k)=\frac{k^{2}}{2} \phi(k), \phi \in L^{2}(\mathbb{R})
$$

The domain of $h$ is $\mathcal{D}(h)=\left\{\left.\phi \in L^{2}(\mathbb{R})\left|\int_{\mathbb{R}} k^{4}\right|(\mathcal{F} \phi)(k)\right|^{2} d k<\infty\right\}$. In the continuous model, both $Q$ and $h$ are unbounded and we have to consider the domain of $h-E Q$ carefully. The operator $h-E Q$ defined on $\mathcal{D}(h-E Q)=\mathcal{D}(h) \cap \mathcal{D}(Q)$ as

$$
(h-E Q) \phi=h \phi-E Q \phi, \phi \in \mathcal{D}(h-E Q),
$$

is essentially self-adjoint [20], that is, the closure of it (we denote $h_{E}$ ) is a self-adjoint operator.

As in Section 2, one can introduce the dissipative dynamics. The NESS is a quasi-free state generated by the following operator on $L^{2}(\mathbb{R})$ :

$$
2 \lambda \int_{0}^{\infty} e^{-2 \lambda s} e^{-i s h_{E}} f_{\beta, \mu}(h) e^{i s h_{E}} d s .
$$

As the calculation in Section 3 shows, this operator is the multiplication operator on momentum space of the function:

$$
\left(R_{\beta, \mu}^{\lambda, E}\right)(k)=2 \lambda \int_{0}^{\infty} e^{-2 \lambda s} f_{\beta, \mu}(\epsilon(k+s E)) d s .
$$

Here we calculate the current density as the integral of the momentum $k$ (this corresponds to our definition of current in lattice models ).

$$
\begin{aligned}
j^{\lambda}(E ; \beta, \mu) & =\int_{\mathbb{R}} k\left(2 \lambda \int_{0}^{\infty} e^{-2 \lambda s} f_{\beta, \mu}(\epsilon(k+s E)) d s\right) d k \\
& =\int_{\mathbb{R}}\left(2 \lambda \int_{0}^{\infty}(k-s E) e^{-2 \lambda s} d s\right) f_{\beta, \mu}(\epsilon(k)) d k \\
& =\frac{E}{2 \lambda} \int_{\mathbb{R}} \frac{1}{1+e^{\beta\left(k^{2}-\mu\right)}} d k .
\end{aligned}
$$

$\int_{\mathbb{R}} \frac{1}{1+e^{\beta\left(k^{2}-\mu\right)}} d k$ is the density of particles per unit volume, and we denote it by $\rho$. Writing the mass $m$ and the charge $q$ explicitly, the last equation becomes

$$
j^{\lambda}(E ; \beta, \mu)=\frac{1}{2 \lambda} \frac{\rho q^{2}}{m} E .
$$


Noting that $(2 \lambda)^{-1}$ means the relaxation time, this is the same as the formula known as the Drude formula.

\section{References}

1. Kubo, R. Statistical-mechanical theory of irreversible processes. I. General theory and simple applications to magnetic and conduction problems. J. Phys. Soc. Jpn. 1957, 12, 570-586. [CrossRef]

2. Bruneau, L.; De Bievre, S.; Pillet, C.-A. Scattering induced current in a tight-binding band. J. Math. Phys 2011, 52, 022109. [CrossRef]

3. De Roeck, W.; Fröhlich, J.; Schnelli, K. Quantum diffusion with drift and the Einstein relation. I. J. Math. Phys. 2014, 55, 075206. [CrossRef]

4. De Roeck, W.; Fröhlich, J.; Schnelli, K. Quantum diffusion with drift and the Einstein relation. II. J. Math. Phys. 2014, 55, 075207. [CrossRef]

5. Davies, E.B. Irreversible dynamics of infinite fermion systems. Comm. Math. Phys. 1977, 55, 231-258. [CrossRef]

6. Evans, D.E. Completely positive quasi-free maps on the CAR algebra. Comm. Math. Phys. 1979, 70, 53-68. [CrossRef]

7. Fannes, M.; Rocca, F. A class of dissipative evolutions with applications in thermodynamics of fermion systems. J. Math. Phys. 1980, 21, 221-226. [CrossRef]

8. Davies, E.B. Markovian master equations. Comm. Math. Phys. 1974, 39, 91-110. [CrossRef]

9. Aizenman, M.; Graf, G.M. Localization bounds for an electron gas. J. Phys. A 1998, 31, 6783. [CrossRef]

10. Thouless, D.J.; Kohmoto, M.; Nightingale, M.P.; den Nijs, M. Quantized Hall conductance in a two-dimensional periodic potential. Phys. Rev. Lett. 1982, 49, 405. [CrossRef]

11. Bellissard, J.; van Elst, A.; Schulz-Baldes, H. The noncommutative geometry of the quantum Hall effect. J. Math. Phys. 1994, 35, 5373-5451. [CrossRef]

12. Chernoff, P.R. Note on product formulas for operator semigroups. J. Funct. Anal. 1968, 2, 238-242. [CrossRef]

13. Spohn, H.; Lebowitz, J.L. Irreversible Thermodynamics for Quantum Systems Weakly Coupled to Thermal Reservoirs. Adv. Chem. Phys. 1978, 38, 109-142.

14. Ruelle, D. Natural nonequilibrium states in quantum statistical mechanics. J. Stat. Phys. 2000, 98, 57-75. [CrossRef]

15. Jakšić, V.; Pillet, C.-A. Mathematical theory of non-equilibrium quantum statistical mechanics. J. Stat. Phys. 2002, 108, 787-829. [CrossRef]

16. Žnidarič, M. Exact solution for a diffusive nonequilibrium steady state of an open quantum chain. J. Stat. Mech. Theory Exp. 2010, , L05002. [CrossRef]

17. Prosen, T. Open XX Z Spin Chain: Nonequilibrium Steady State and a Strict Bound on Ballistic Transport. Phys. Rev. Lett. 2011, 106, 217206. [CrossRef] [PubMed]

18. Tasaki, S. Nonequilibrium stationary states of noninteracting electrons in a one-dimensional lattice. Chaos Solitons Fractals 2001, 12, 2657-2674. [CrossRef]

19. Aschbacher, W.H.; Pillet, C.-A. Non-equilibrium steady states of the XY chain J. Stat. Phys. 2003, 112, 1153-1175. [CrossRef]

20. Reed, M.; Simon, B. Methods of Modern Mathematical Physics, Vol IV: Analysis of Operators; Academic Press: New York, NY, USA, 1978

(c) 2020 by the authors. Licensee MDPI, Basel, Switzerland. This article is an open access article distributed under the terms and conditions of the Creative Commons Attribution (CC BY) license (http://creativecommons.org/licenses/by/4.0/). 